\title{
Estimation of airframe weight reduction by integration of piezoelectric and guided wave-based structural health monitoring
}

Structural Health Monitoring

\author{
Christoph P Dienel', Hendrik Meyer ${ }^{2}$, Malte Werwer ${ }^{3}$ and \\ Christian Willberg'
}

\begin{abstract}
Current design rules are analyzed and challenged by considering better damage-detection capabilities offered by structure health monitoring systems. The weight-saving potential associated to the integration of such sensing systems is discussed with regards to structural and structure health monitoring system design. Three prospective scenarios are analyzed and considered in a specific use case. According to the most promising scenario (i.e. robust detectability of damages larger than $300 \mathrm{~mm}^{2}$ ), structural weight reductions of approximately $9 \%$ can be achieved. Considering the weight added by the structure health monitoring system, effective weight savings in the order of $5 \%$ are achievable. Although this potential is rather modest, applying structure health monitoring systems on structures mainly driven by the damage tolerance criterion is expected to provide reductions far beyond $5 \%$.
\end{abstract}

\section{Keywords}

Airframe, weight reduction, design rules, system weight, carbon fiber reinforced plastic, structure health monitoring, integrated vehicle health management

\section{Introduction}

Since the late 1970 s, most primary aircraft structure is designed according to the damage tolerance philosophy. It is intended to ensure that structures, damaged due to fatigue, corrosion, or accidental damage, are capable of withstanding reasonable loads without failure or excessive structural deformation until the damage is detected. ${ }^{1}$ For composite structures, the relevant design driving damage types can be narrowed down to accidental damage, as the others are highly unlikely to occur. $^{2}$ In considering visual inspection as the currently appointed damage-detection method, allowable damage limits (ADLs) - generally described in terms of dent depth or projected damage area - are defined for different airframe parts. Damage equal to or larger than this threshold is assumed to reduce residual strength and must therefore be reliably detected by scheduled or directed field inspections. ${ }^{3}$ The definition of ADLs is driven by its detectability, which in turn takes into account lighting conditions, cleanliness of the surfaces, paint color, and so on. These conditions may not always be ideal for visual damage detection, thus demanding for rather conservative thresholds.

It is based on the empirically substantiated relation between damage size and residual strength that airframe structures are sized. ${ }^{4}$ Figure 1 is a schematic of that relation and can be understood as a conservative curve (B-value) fit through experimental data points.

According to the damage tolerance philosophy, structures with damages up to ADL are required to ensure design ultimate load (DUL) capability. The critical damage threshold (CDT) describes defects that

\footnotetext{
'Institute of Composite Structures and Adaptive Systems, German Aerospace Center, Braunschweig, Germany

${ }^{2}$ Institute of Maintenance, Repair and Overhaul, German Aerospace Center, Hamburg, Germany

${ }^{3}$ Airbus Operations GmbH, Hamburg, Germany
}

\section{Corresponding author:}

Christoph P Dienel, Institute of Composite Structures and Adaptive Systems, German Aerospace Center, Lilienthalplatz 8, 38108

Braunschweig, Germany.

Email: christoph.dienel@dlr.de 


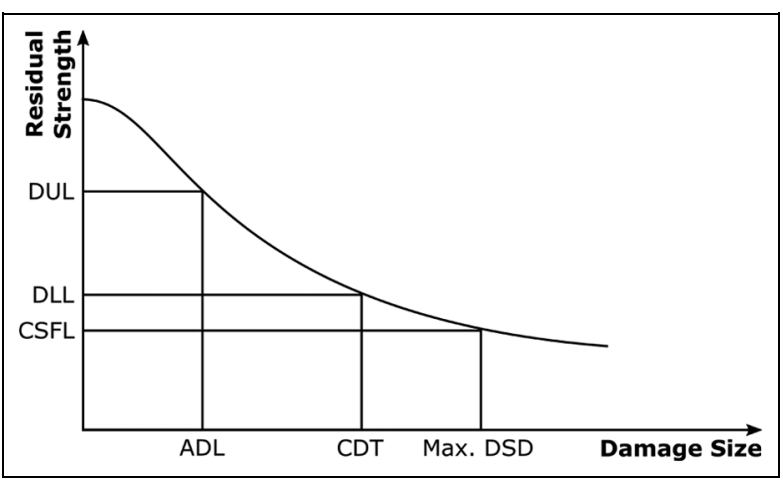

Figure I. Damage threshold and according allowable load derivation from experimental results.

cause the residual strength to drop to design limit load (DLL). The residual strength of a structure with a maximum discrete source damage (max. DSD) is referred to as continued safe flight and landing load (CSFL). ${ }^{5}$

Postulated that the integration of structure health monitoring (SHM) systems can enable the detection of damage below ADL, two promising scenarios are offered as follows:

1. Since current scheduled inspections are also determined by the limited damage detectability, ${ }^{3}$ by keeping current design allowables, a robust method for detecting damage below ADL would result in an extension of inspection intervals toward demand-oriented maintenance.

2. In adjusting the design allowables to smaller ADLs, a better material exploitation could be achieved, resulting in thinner and thus lighter structures.

This article is focused on the second scenario and presents an estimation of the structural weight reduction that can potentially be achieved by the integration of SHM systems for on-line and on-board monitoring. It is worth noting though, that to date, no off-the-shelf systems capable of robustly detecting small accidental damage on composite structures exist.

Different kinds of SHM systems have been proposed for these purposes during the past decade. ${ }^{6}$ Among them, ultrasonic guided wave-based SHM systems are a very promising approach to detect impact damages and to evaluate the resulting damage size. ${ }^{7} \mathrm{Su}$ and $\mathrm{Ye}^{8}$ give an overview about the several steps needed utilizing a guided wave-based SHM system. Several systems are in development and to some regard commercially available. ${ }^{9-11}$ The wavelength of ultrasonic waves depends on the wave mode and the excitation frequency used for the monitoring system. ${ }^{12}$ On the one side, a smaller wavelength is capable of detecting smaller flaws. On the other side, the size of the monitored region is reduced and more sensors are required to cover the same region as compared to larger wavelengths. For this purpose, piezoelectric (PZT) sensors have shown to be adequate $^{13}$ and are considered to be the sensors in this study.

While addressing a fictive SHM system regarding its sensing capabilities, the contribution of such an integration to the air vehicle fuel consumption can be estimated based on currently available data for the selected use case. $^{14,15}$

\section{Approach}

The approach adopted to estimate the weight reduction potential due to the integration of SHM systems consists in adjusting the damage tolerance criterion to satisfy new ADLs, smaller than the barely visible impact damage (BVID), assumed to be detectable by prospective SHM systems.

Especially for damage tolerance-driven design, this adjustment leads to structural thickness reductions. However, since many sizing criteria generally apply to the same structure, this may lead to another sizing criterion becoming dimensioning instead. Therefore, the weight reduction potential estimation is conducted under the simultaneous consideration of the relevant sizing criteria, presented in the following section.

From the sizing criteria considered, allowable strain envelopes are derived for different scenarios, ranging from the conventional to prospective ADLs. For each scenario, a thickness histogram is created to visualize the skin thickness re-assignment, and finally, the structural weight is calculated.

For the system weight added to the structural weight, the quantity of sensors is estimated. Parameters such as weight and power consumption are derived from the corresponding sensor data sheets. Also the wires' weight for power supply and data transfer will be calculated depending on the power demand and the estimated data transmission rate. Finally, the change in weight, resulting from the new design and SHM system wiring, is combined with the electrical power demand in the Breguet-formula ${ }^{16}$ in order to derive the fuel consumption change.

\section{Sizing criteria for the structural design}

Depending on the loading type, joint configuration, design features, and the susceptibility to accidental damage, different sizing criteria may become dimensioning for the structure. In the following, a selection of a few important criteria is introduced. The design allowables resulting from these sizing rules are expressed in terms of nominal strain for the sake of 
comparability. Furthermore, the critical strain is additionally reduced by a knock-down factor $(K D F)$ that accounts for the scatter in experimentally determined material properties (B-value) and for environmentbased effects. The latter component, however, applies only to matrix failure-dominated sizing criteria.

\section{Stability}

Thin flat structures loaded in compression are expected to experience stability failure (buckling) at a critical load. Depending on the boundary conditions applied to the structure, the layup and material properties as well as the geometric parameters, the critical buckling strain $\varepsilon_{\text {stab }}$ can be re-written from the analytical expression given by Hwang and $\mathrm{Lee}^{17}$ as

$$
\varepsilon_{\text {stab }}=\frac{k \cdot \pi^{2} \cdot \sqrt{D_{11} \cdot D_{22}}}{w^{2} \cdot t \cdot E_{x}} \cdot K D F \cdot 10^{6}
$$

The buckling coefficient $k$ accounts for the buckling field's aspect ratio, adjusted by the laminate's anisotropy, being defined as

$$
k=\frac{m^{2}}{R^{2}}+\frac{R^{2}}{m^{2}}+2 \cdot \eta
$$

considering

$$
R=\left(\frac{D_{22}}{D_{11}}\right)^{\frac{1}{4}} \cdot \frac{a}{b} \quad \text { and } \quad \eta=\frac{D_{12}+2 \cdot D_{66}}{\sqrt{D_{11} \cdot D_{22}}}
$$

In view of the use case being introduced later, the aspect ratio $a / b$ of the buckling field is set to 1 . Following the conservative assumption of all buckling field edges being simply supported, the parameter $m$ can be set to 1 too. The coefficients $D_{i j}$ are the plate's flexural rigidities. The remaining parameters in equation (1) represent the buckling field's width $(w)$, its thickness $(t)$ and the laminate Young's modulus in loading direction $\left(E_{x}\right)$. As being a fiber-dominated failure mode, the effective $K D F$ accounts only for the experimental scatter at the material characterization.

By considering the thickness dependency of the flexural rigidity as given by equation (4), a quadratic relation between the critical buckling strain $\varepsilon_{\text {stab }}$ and the skin thickness $t$ is found. Hereby, the index $d=x, y$ represents the main laminate directions

$$
D_{i i}=\frac{E_{d} \cdot t^{3}}{12 \cdot\left(1-\nu_{x y} \cdot \nu_{y x}\right)}
$$

\section{Damage tolerance}

Most aircraft structures are prone to accidental damage. However, predicting the residual strength deterministically for a given impact energy or damage size is not yet reliably solved. Therefore, aircraft manufacturers base their residual strength estimates on results acquired in extensive experimental campaigns. From the obtained measurement points, a mathematical relation between residual strength and the laminate thickness is derived by determining the best-fitting curve. This relation is established for specimens tested under compression load, since this is the most critical loading case. ${ }^{4,18}$

To the authors knowledge, neither deterministic nor experimentally derived expressions relating residual strain to influencing parameters such as laminate thickness, layup, and damage area have been published so far. Therefore, the authors recurred to results obtained from an extensive testing campaign conducted at Airbus. The empirical expression describing that relation reads (equation (5))

$$
\varepsilon_{\mathrm{DT}}^{\mathrm{comp}}=p \cdot A_{d}^{-q \cdot f(t)} \cdot C_{\text {Layup }} \cdot K D F
$$

This equation reflects the best-fitting curve through the experimental results. Residual strain $\varepsilon_{\mathrm{DT}}^{\text {comp }}$ is described as a function of the projected delamination area $A_{d}$, the laminate thickness $t$, and the layup's ortho-

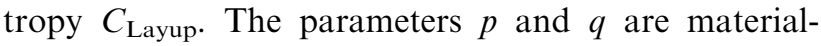
specific parameters. The function $f(t)$ describing the effect of thickness on the residual strain and the layup parameter $C_{\text {Layup }}$ accounting for orthotropy are defined as

$$
f(t)=a \cdot t^{b}+c
$$

and

$$
C_{\text {Layup }}=\frac{1}{16} \cdot\left(19-\frac{E_{x}}{G_{x y}}\right)
$$

The coefficients $a, b$, and $c$ are curve fitting parameters, while $G_{x y}$ represents the laminate's shear modulus. Under compression load, the behavior of impact-damaged structures is strongly affected by matrix failure. Therefore, the environmental knock-down component is considered along with the $\mathrm{B}$-value in $K D F$.

For simplification, it is assumed that the projected delamination area $A_{d}$ is constant for a given impact threat, regardless of the laminate thickness. This is a rather conservative approach, as thinner laminates are more compliant in transversal direction, thus absorbing more elastic (reversible) energy and suffering less damage. $^{19,20}$

\section{Bearing strength}

Bolted joints are widely used in joining composite airframe parts and for structural repairs. This joining 
method results in stress concentrations in the immediate bolt (or rivet) vicinity. Bolt failure can easily be prevented by choosing the right bolt material and geometry. However, mating parts can suffer different failure modes, depending on a series of geometrical and layup parameters. Large diameter-to-width ratio can lead to net-tension failure; conversely, a small ratio favors bearing failure; which also occurs at low laminate thickness to bolt diameter ratios. Highly orthotropic laminates and short end distance may cause shear-out failure. Cleavage can be expected for short end distances and too few transversal plies. ${ }^{21}$ Yet, these failure modes can also be prevented from occurring by properly choosing the geometric parameters.

Since the bearing strength, obtained by experimental testing, determines the bolted joints parameters, an allowable strain can be set as a requirement. In this analysis, the bearing strain allowable $\varepsilon_{\mathrm{BS}}$ is therefore fixed at $5000 \mu \varepsilon$, which is a commonly applied threshold.

\section{Maximum strain}

Composite airframe structures generally fail due to other phenomena before reaching the maximum material strain capability. Nevertheless, and for the sake of completeness, the maximum strain criterion is considered here too. The strain at which first ply failure sets in, depends on the layup and the loading condition. The general expression given by equation (8) is the Hook's law, reduced by the $K D F$ and converted to micro-strain $(\mu \varepsilon)$ units. The stiffness $S_{i i}$ and the strength $\sigma_{i i}$ are the elasticity moduli and strength in different directions, respectively (11: in fiber direction, 22: perpendicular to the fiber, 12: in-plane shear, + : tension, -: compression)

$$
\varepsilon_{i i}=\frac{\sigma_{i i}}{S_{i i}} \cdot K D F \cdot 10^{6}
$$

Since a typical airframe layup is generally composed by plies in $0^{\circ}, 90^{\circ}, 45^{\circ}$, and $-45^{\circ}$, the first ply failure is predicted to occur, once the strain along one of the main laminate axis has reached the minimum critical strain $\varepsilon_{\mathrm{MS}}$

$$
\varepsilon_{\mathrm{MS}}=\min \left\{\varepsilon_{11}^{+}, \varepsilon_{11}^{-}, \varepsilon_{22}^{+}, \varepsilon_{22}^{-}, \varepsilon_{12}\right\}
$$

\section{Structural weight-saving potential}

In the preliminary design phase, airframe structure weight estimations are understood as rough calculations, based on a variety of simplifying assumptions. These assumptions are applied on both designs: the conventional and the new, resulting from the integration of SHM systems. Thus, a comparison between both designs is allowed and the potential can be identified by comparing the respective structural weights. It is assumed that

- All sizing criteria mentioned above apply to the same structure simultaneously;

- Each airframe structure considered is sized by the lowest strain allowable resulting from the sizing criteria above;

- The structure is loaded in-plane under uniaxial conditions;

- Sizing criteria are applied only to skins; it is postulated that stringers and frames fail at higher strains;

- The stringer pitch is set to a constant value of $130 \mathrm{~mm}$;

- Only skins are resized due to the integration of SHM systems;

- Thickness adjustments are continuous and disregard the single ply thickness;

- In-plane stiffness is kept constant regardless of the assigned thickness;

- The reference damage size of $600 \mathrm{~mm}^{2}$ corresponds to a BVID that would be detectable by visual inspection according to this method.

- As an SHM system, a guided wave-based system utilizing piezoceramic sensors and actuators is considered.

The structural weight obtained by considering SHM as the alternative damage-detection method to the current visual inspection method is computed for three different scenarios, where the SHM system is assumed to reliably detect damage areas of 300,400 , and $500 \mathrm{~mm}^{2}$.

In Figure 2, the allowable strains are plotted as functions of the skin thickness considering all sizing criteria mentioned above. Dashed curves represent individual sizing rules, whereas solid lines illustrate the minimum

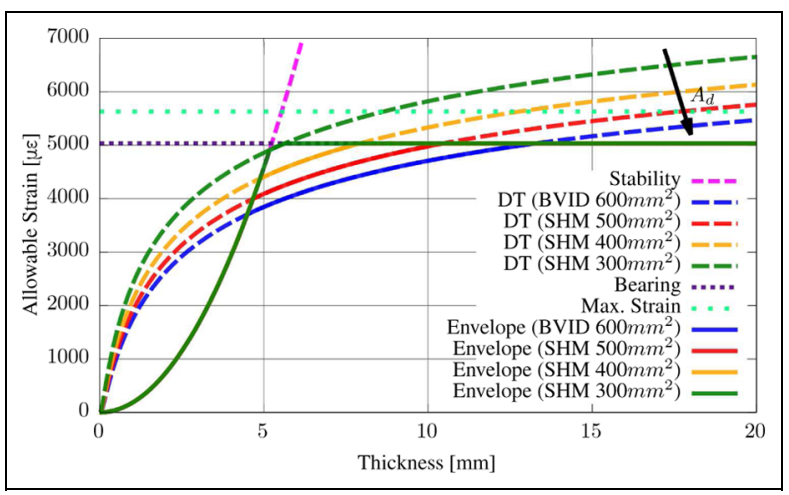

Figure 2. Allowable strain versus skin thickness for different sizing criteria. 
allowable strain envelopes for this design and the assumed SHM scenarios. The envelopes differ only in the damage tolerance-driven domain $t \in[4.5,13.0]$. As indicated by the arrow, the larger the detectable damage $A_{d}$, the lower the allowable strain.

The difference between the BVID envelope and the SHM envelopes are indicators of the weight-saving potential presumably achievable by SHM system implementation. For instance, the allowable strain for a 10mm skin, according to the BVID criterion, amounts to $4710 \mu \varepsilon$. In keeping this allowable strain, thickness could be reduced to 8.0,6.2, and $5.1 \mathrm{~mm}$ for the SHM scenarios 500, 400, and $300 \mathrm{~mm}^{2}$, respectively. In other words, the weight-saving potential is $20 \%, 38 \%$, and $49 \%$, accordingly.

\section{SHM system layout}

In the following section, the SHM system architecture, the associated added weight, the power demand, and the data bus architecture are addressed.

\section{Sensor}

As reference for the weight and power consumption estimation, a PZT sensor (Series 740) from PCB Piezotronics Inc, MTS System Corporation ${ }^{22}$ has been selected, for which a technical data sheet is available, while for many other sensors, typically used for SHM research, only the material is known. ${ }^{23}$ Besides that, such sensors are neither industrialized nor adequate for permanent implementation in aircrafts, since they are only available without housing and connectors. It is noted too, that although the reference sensor is designed for sensing rather than for exciting ultrasonic waves, it is assumed that the properties regarding weight and power consumption of both sensor types are comparable and suitable for a system assessment.

The PZT sensors with a frequency ranging from 0.5 to $100,000 \mathrm{~Hz}$ can be used for impact event monitoring and damage size estimation. The sensors contain builtin electronics and require a power/signal conditioner. The temperature compensation is provided. The sensor weight is $0.5 \mathrm{~g}$, and the adhesive mass for sensor placement is regarded as negligible. The signal/power conditioner requires $2-20 \mathrm{~mA}$, operating from a $18-30 \mathrm{~V}$ (DC) power source, with a weight of $40 \mathrm{~g}$. It is assumed that 10 sensors are required to cover a $1 \mathrm{~m}^{2}$ generic panel. For this number of sensors, satisfactorily results have already been achieved in the context of optimized sensor placement on flat plates for defect sizes smaller than the most ambitions scenario considered in this study. ${ }^{24}$
The data amount produced by sensors depends on sampling frequency. In general, the data rate $C$ can be calculated using the following equation

$$
C=n_{\mathrm{ch}} \cdot B \cdot R E S
$$

where $B$ is the sampling frequency, RES is the resolution, and $n_{\mathrm{ch}}$ is the number of channels. The typical resolution is 12 bit. $^{25}$ The systems with high sensitivity can require resolution of 24 or 32 bit.

\section{Usable bandwidth}

Bandwidth refers to the total amount of data that can theoretically be transferred through the bus in a given period of time. Due to necessity for physical synchronization and message heading, which is provided by $D_{\text {protocol }}$ and $D_{\text {overhead, a portion of the theoretical }}$ bandwidth $B_{\text {theo }}$ is needed for performing these tasks. The amount of data that can be actually transmitted via bus, that is, the usable bandwidth $B_{\text {usable, }}$, is expressed as a percentage of payload data $D_{\text {pay }}$ in bites divided by the total number of bites in a message. ${ }^{26}$ The usable bandwidth $B_{\text {usable }}$ is given by

$$
B_{\text {usable }}=\frac{D_{\text {pay }}}{D_{\text {pay }}+D_{\text {protocol }}+D_{\text {overhead }}} \cdot B_{\text {theo }}
$$

\section{Distributed integrated modular avionics architecture}

For the approach presented in this article, a distributed integrated modular avionics (DIMA) is selected, which is a typical architecture to both composite aircrafts Airbus A350 and Boeing 787 (Figure 3).

DIMA architecture provides computation hardware in the proximity of sensors and actuators, thus resulting in cable weight reduction and faster system response. Systems dedicated to cockpit, cabin, flight control, engines, fuel, and so on, are separated into integration areas for data condensation. Data concentrators condense and convert sensor and actuator data into a common digital format and then transfer these data to the DIMA modules using data buses (Figure 3). In addition, they also perform simple logic operations and issue alarms. ${ }^{29,30}$

The quantity of cables for power supply depends on the number of sensors $n_{\text {sensor }}$ and instruments, such as sensing system interrogators. To reduce the cable quantity needed for data transfer, the analog signals can be locally converted into digital data, which are then transferred via serial data buses. Thus, the required data bus number $n_{\text {bus }}$ per aircraft structure or component can be calculated by 


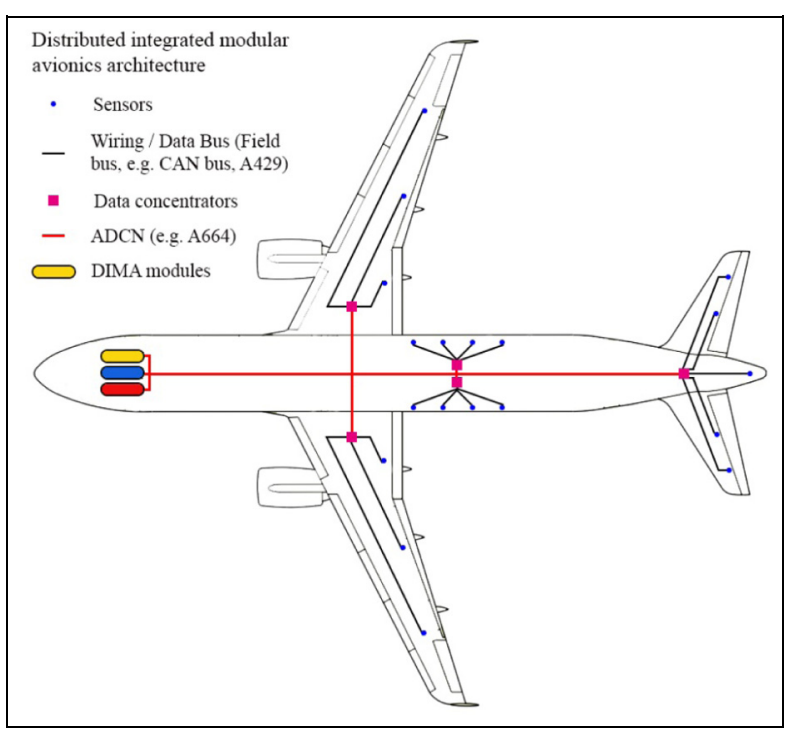

Figure 3. Distributed integrated modular avionics architecture based on Annighoefer et al. ${ }^{26}$ and Eckman. ${ }^{27}$

Aircraft source: Airbus. ${ }^{28}$

$$
n_{\text {bus }}=\frac{c \cdot n_{\text {sensor }}}{B_{\text {usable }}}
$$

where $c$ is the data rate of the selected sensor type.

The cable length required for electrical power supply and data transfer for the SHM network depends on the aircraft's geometry. For the cable length calculation, the following assumptions are made regarding cable distribution (Figure 4).
The cable length for the vertical tail plane (VTP)

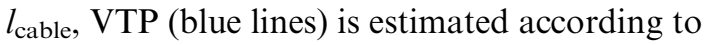

$$
l_{\text {cable }, \mathrm{VTP}}=\left(\frac{1}{3} \cdot h_{\mathrm{VTP}}+l_{\text {fus }}\right) \cdot k_{\text {cable }}
$$

where $k_{\text {cable }}=1.2$ is a coefficient accounting for indirect cable paths, $h_{\mathrm{VTP}}$ is the VTP height, and $l_{\text {fus }}$ is the fuselage length.

It is assumed that for metallic airframes, the power supply cable requires one conductor, whereas for composite airframes, the power supply cable requires two conductors, thus double the calculated length in equation (13) is required.

For DIMA (Figure 5) architectures, it is assumed the areas marked with green lines are equipped with field buses, whereas the red lines marked areas with ARINC 664 or ARINC 629.

\section{Wire size selection}

A standard known as the American Wire Gauge (AWG) is used for wire manufacturing. The Federal Aviation Administration (FAA) ${ }^{31}$ describes factors to be considered in selecting the wire size for electric power distribution. A conductor chart for continuous flow $^{31}$ can be used to select the proper conductor size. In general, the wire size must be sufficient to prevent cable overheating and voltage drop while conducting the required current. According to the FAA, ${ }^{31}$ the smallest allowable single wire size is 22 AWG. The

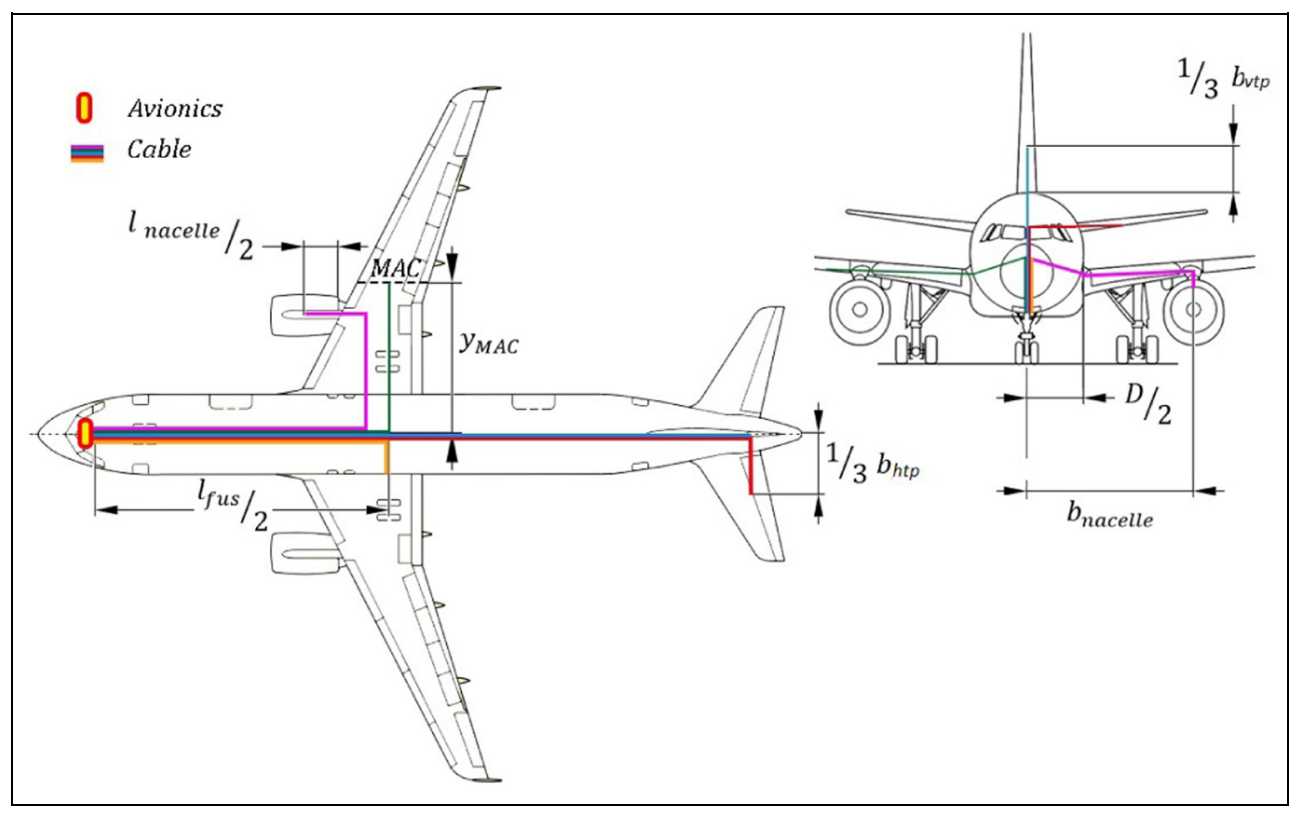

Figure 4. Cable distribution Aircraft source: Airbus. ${ }^{28}$ 


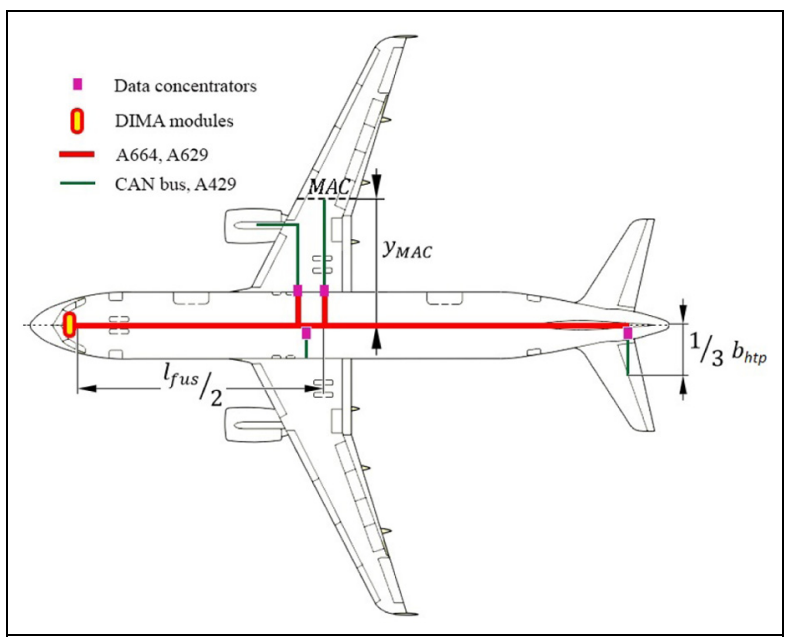

Figure 5. Assumption of DIMA architecture data buses distribution.

Aircraft source: Airbus ${ }^{28}$

circuit voltage of $28 \mathrm{~V}$ (DC) as mentioned in the conductor chart is suitable for all sensor types. It is assumed that all sensors are parallel connected, thus the resulting power is obtained by multiplication of the single sensor power consumption by the number of sensors. For the cable amount estimation, a wire size of 20 AWG (650 kg. km ${ }^{-1}$ maximum weight) is required, as the 22 AWG wire would not satisfy the cable length requirements. For the given voltage of $28 \mathrm{~V}$ (DC), a maximum of 50 PZT sensors for impact monitoring can be powered by one wire. The cable weight for the parallel connection implementation of sensors is negligible.

\section{Cable weight}

The cable weight $w_{\text {cable }}$ for power supply and data transfer can be calculated using the following formula

$$
w_{\text {cable }}=l_{\text {cable }} \cdot w_{\text {spez, cable }}
$$

where $l_{\text {cable }}$ is the length and $w_{\text {spez,cable }}$ is the specific cable weight.

The specific cable weight for different cables is provided in Table 1.

\section{Fuel consumption}

Flight performance can be expressed by Breguet range equation. ${ }^{16}$ Considering aircraft parameters such as weight, wing area, and engine type, the maximum total range for given environmental conditions can be calculated. Under the assumption of constant flight velocity, lift coefficient, and specific fuel consumption (SFC), the following expressions apply:
Table I. Specific weight for different cable types (according to Nexans $^{32}$ and Elettrotekkabel S.p. $A^{33}$ ).

\begin{tabular}{lll}
\hline Cable type & $\begin{array}{l}w_{\text {spez, cable }} \\
\left(\mathrm{kg} \cdot \mathrm{km}^{-1}\right)\end{array}$ & Bus type \\
\hline ASNE 0272 AWG 22 & 12.70 & ARINC 429 \\
STUDY I2496 AWG 24 & 37 & ARINC 629 \\
ABS I503 KD 24 AWG 24 & 40.28 & ARINC 664 \\
CAN bus AWG 22 & 21.80 & CAN Bus \\
\hline
\end{tabular}

Table 2. Correlation between the electrical power drain and variations in specific fuel consumption. ${ }^{35}$

\begin{tabular}{lll}
\hline Flight phase & Thrust class $(\mathrm{kN})$ & $\Delta S F C$ for $100 \mathrm{~kW}(\%)$ \\
\hline Climb & 150 & $0.4-0.8$ \\
Cruise & 150 & $0.7-1.5$ \\
Descent & 150 & $3.0-7.0$ \\
Climb & 300 & $0.3-0.5$ \\
Cruise & 300 & $0.6-1.1$ \\
Descent & 300 & $3.0-5.0$ \\
\hline
\end{tabular}

SFC: specific fuel consumption.

Lift

$$
L=m \cdot g=\frac{1}{2} \cdot \rho \cdot V^{2} \cdot C_{\mathrm{L}} \cdot S_{\mathrm{ref}}
$$

where $L$ is the aerodynamic lift, $m$ is the aircraft weight, $\rho$ is the air density, $V$ is the flight velocity, $C_{\mathrm{L}}$ is the lift coefficient, $S_{\text {ref }}$ is the wing area, and $g$ is the acceleration due to gravity.

Thrust

$$
T=D=\frac{1}{2} \cdot \rho \cdot V^{2} \cdot C_{\mathrm{D}} \cdot S_{\mathrm{ref}}
$$

where $T$ is the thrust, $D$ is the drag, $C_{\mathrm{D}}$ is the drag coefficient.

Breguet range

$$
R=\frac{V \cdot L}{g \cdot S F C \cdot D} \cdot \ln \frac{m_{\text {initial }}}{m_{\text {final }}}
$$

where $R$ is the maximum range, $m_{\text {initial }} / m_{\text {final }}$ is the weight ratio (initial over final), and $S F C$ is the specific fuel consumption. ${ }^{16}$ Typical $L / D$ ratios for commercial aircrafts range from 18 to 22 for the cruise flight phase. ${ }^{34}$

Based on the correlations given by Dollmayer and Carl, ${ }^{35}$ sensor power requirement and thus SHM electrical energy consumption can be expressed in terms of SFC. Table 2 depicts two engine classes and the respective SFC change for a $100 \mathrm{~kW}$ energy drain at different flight phases. 


\section{Use case: the VTP skin}

\section{Structural design}

In order to provide a figure for the weight-saving potential, a reference structure and the respective thickness map (i.e. a thickness histogram) is required. In this article, one VTP skin is considered as an exemplary use case. Although being a stiffened shell, only the skin is subjected to thickness adjustments. Moreover, all the assumptions listed in section "Structural weight-saving potential" apply to the structure considered in this section.

The VTP skin design of a wide-body aircraft is mainly driven by stability (in this example, $87 \%$ regarding the skin surface). However, some regions' design is determined by the damage tolerance requirement. It can be depicted from the BVID envelope in Figure 2 that for skin thickness below $13 \mathrm{~mm}$, design is determined either by the stability or by the damage tolerance criteria.

In Figure 6, the structural weight for one VTP skin is broken down according to the skin thickness for the conventional design, ranging from 1.84 to $10.12 \mathrm{~mm}$. In spite of covering the majority of the skin surface $(87 \%)$, the chart confirms that the structural mass sized by the stability criterion is rather low (only $59 \%$ ). The remaining structural design is dominated by the damage tolerance requirement. Note that the calculated structure thickness does not reflect a realistic layup, where the laminate thickness is always scaled by integer multiples of the single ply thickness. Instead, the abscissa values are discretized in $0.02 \mathrm{~mm}$ increments in order to focus on the design-based weight reduction potential, instead of focusing on manufacturing issues. Abscissa bars with $0 \mathrm{~kg}$ are excluded from the bar charts below.

Considering an SHM system capable of detecting damage areas slightly smaller than those detected by the current visual inspection method, skin thickness is slightly reduced in the damage tolerance domain. The new VTP skin thickness range $1.84-8.12 \mathrm{~mm}$ results in a weight reduction of approximately $5.1 \%$ (Figure 7).

In the second SHM scenario, damage above $400 \mathrm{~mm}^{2}$ is assumed to be reliably detectable. Further thickness reductions are achieved for the damage tolerance-driven skin regions. The thickness now extends over the range $1.84-6.26 \mathrm{~mm}$, causing the skin weight to drop by $8.4 \%$ from the reference design (Figure 8).

Finally, damage size above $300 \mathrm{~mm}^{2}$ is assumed to be detectable with prospective SHM systems. In this scenario, the skin thickness range is now narrowed down to 1.84-5.06, leading to a weight reduction of $9.2 \%$ compared to the reference (Figure 9). According to Figure 2, a higher damage resolution by the inspection system would be of no value, since the damage tolerance sizing criterion would not apply anymore.

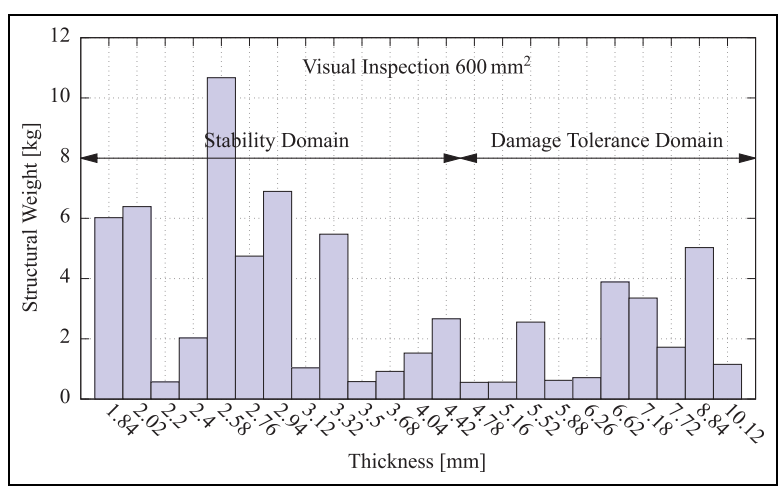

Figure 6. Weight distribution per skin thickness by considering the conventional inspection method with $600 \mathrm{~mm}^{2}$ detectable damage size.

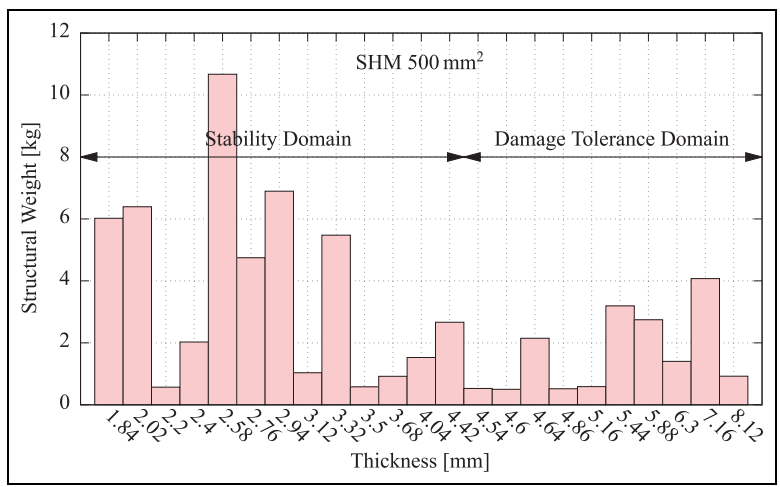

Figure 7. Weight distribution per skin thickness for an SHM system with $500 \mathrm{~mm}^{2}$ detectable damage size.

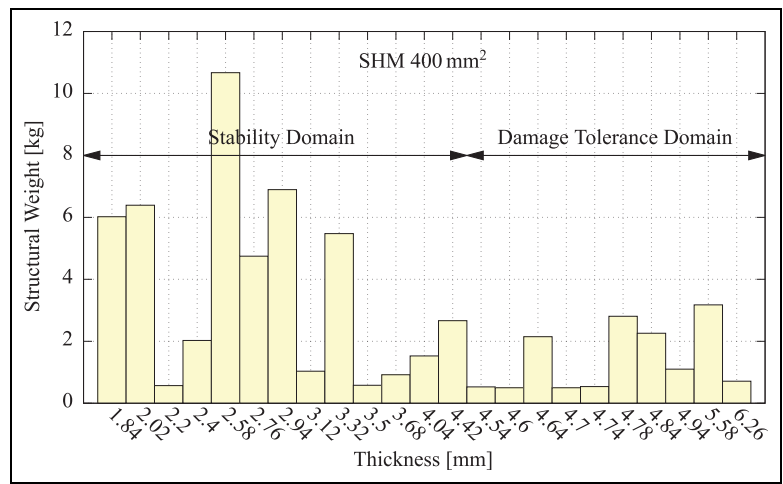

Figure 8. Weight distribution per skin thickness for an SHM system with $400 \mathrm{~mm}^{2}$ detectable damage size.

\section{SHM system design}

For the damage tolerance driven surface $(13 \%$ of the VTP skin surface), an SHM system is designed, which introduces 10 PZT sensors per square meter. This adds 


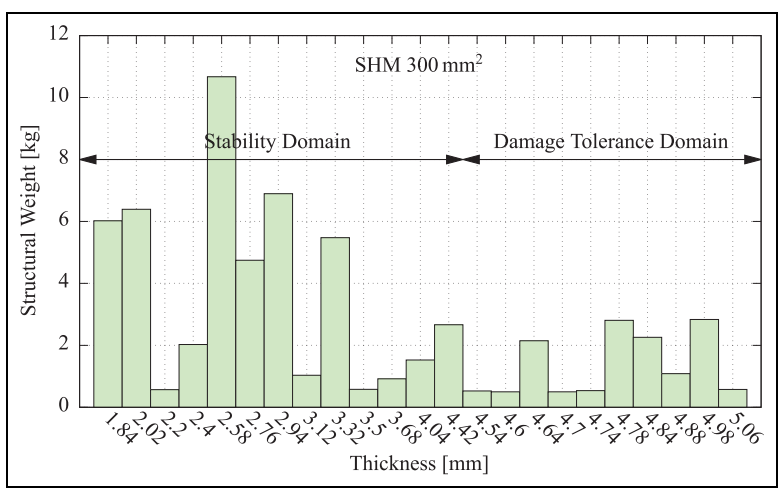

Figure 9. Weight distribution per skin thickness for an SHM system with $300 \mathrm{~mm}^{2}$ detectable damage size.

an additional weight of $1.0 \%$ to the reference design. The power supply lines to these sensors adds further $1.6 \%$ weight. The ARINC 429 data bus system is preferable as the lightest solution for a small monitored area and the low number of sensors required in this use case, yielding additional $1.6 \%$ weight. The resulting mass increase for the SHM system amounts $4.2 \%$.

Another factor to be considered is the power consumption of such a system. For the number of sensors required, the power consumption for the monitored area would be $3.6 \mathrm{~W}$, according to the manufacturer's data sheet. ${ }^{22}$ Due to the small number of sensing elements, the overall power consumption is that low, that in the worst case listed in Table 2 (descent, $300 \mathrm{kN}$ thrust class, $\triangle S F C$ change $5 \%$ per $100 \mathrm{~kW}$ ) the SFC change would amount $1.8 \times 10^{-4} \%$. Although being negligible in this use case, the contribution of a full coverage SHM system to the overall SFC is expected to exert a relevant impact.

Finally, the implementation of an SHM system contributes to reduced or changed maintenance schedule. However, in this use case, this advantage is nullified by the need for further visual inspection due to only partial structure monitoring.

\section{Weight-saving potential}

Considering the three prospective SHM scenarios mentioned above, three weight-saving potential figures can be derived by regarding new structural and SHM system designs as summarized in Table 3.

\section{Fuel-saving potential}

Considering the SFC change $\left(1.8 \times 10^{-4} \%\right)$ calculated for the SHM system adopted in this use case, and the maximum estimated structural weight reduction, as mentioned above $(5.0 \%)$, the potential fuel saving can
Table 3. Weight-saving potential for each scenario considered in this use case.

\begin{tabular}{llll}
\hline $\begin{array}{l}\text { Detectable } \\
\text { damage size } \\
\left(\mathrm{mm}^{2}\right)\end{array}$ & $\begin{array}{l}\text { Wy re-design } \\
(\%)\end{array}$ & $\begin{array}{l}\text { by SHM system } \\
(\%)\end{array}$ & $\begin{array}{l}\text { Total } \\
(\%)\end{array}$ \\
\hline 300 & -9.2 & +4.2 & -5.0 \\
400 & -8.4 & +4.2 & -4.2 \\
500 & -5.1 & +4.2 & -0.9 \\
\hline
\end{tabular}

SHM: structural health monitoring.

be quantified. Applying equation (17) to a standard mission $\left(\mathrm{R}=10,000 \mathrm{~km}, \quad g \approx 9.75 \mathrm{~m} \mathrm{~s}^{-2}, \quad\right.$ and $\left.V=900 \mathrm{~km} \mathrm{~h}^{-1}\right)$, and a modern long-range aircraft $\left(L / D=21\right.$ and maximum zero-fuel weight $m_{\text {final }}=$ $195,700 \mathrm{~kg}$ ), a negligible fuel increase of approximately $0.017 \%$ is obtained.

By extrapolating the structural weight-saving potential calculated for the VTP skin to a long-range aircraft (airframe weight: $80,000 \mathrm{~kg}$ ), considering the improved detection capability, the additional equipment weight, and the associated power consumption of the most promising SHM scenario considered, fuel consumption could be reduced by roughly $1.8 \%$.

\section{Conclusion}

The probability of damage detection is an important driver for both: structural design and maintenance scheduling. From recent advances in SHM systems, it can be projected, that smaller damages than those detectable by visual inspection, as considered in current structural design, can be reliably detected in the near future. The approach presented in this article allows a first estimation of the weight reduction achievable by the implementation of such systems.

To demonstrate the weight-saving potential, the VTP skin was selected as the use case. For that structure, weight reductions up to $5 \%$ are achievable by the integration of an SHM system with a $300-\mathrm{mm}^{2}$ damage size detection capability. This fraction may seem disappointingly low. However, it must be considered that this particular structure is mainly sized by the stability criterion ( $87 \%$ of the area). Since only damage tolerance-driven structures benefit from the SHM system integration, only a slight weight reduction is expected for the VTP skin. Other regions of the airframe structure driven by the damage tolerance criterion are expected to provide more significant weight reductions. This result suggests that the integration of SHM capability should mainly focus on damage tolerance-driven structures. 
Projecting the findings regarding the VTP skin to the entire aircraft structure, significant fuel consumption reductions beyond $1.8 \%$ could be attained.

\section{Declaration of conflicting interests}

The author(s) declared no potential conflicts of interest with respect to the research, authorship, and/or publication of this article.

\section{Funding}

The author(s) disclosed receipt of the following financial support for the research, authorship, and/or publication of this article: This study was performed within the EU-Horizon 2020 Project DEMETER (Grant Agreement No. 685704).

\section{ORCID iD}

Christian Willberg (iD https://orcid.org/0000-0003-2433-9183

\section{References}

1. FAA. AC 25.571-1D-damage tolerance and fatigue evaluation of structure document information. Washington, DC: FAA, 2011.

2. Soutis C. Carbon fiber reinforced plastics in aircraft construction. Mater Sci Eng A 2005; 412(1): 171-176.

3. EASA. Acceptable means of compliance 20-29 / Amendment 6. Cologne: EASA, 2010.

4. Faivre V and Morteau E. Damage tolerant composite fuselage sizing. FAST 2011; 48: 10-16.

5. Razi $\mathrm{H}$ and Ward S. Principles for achieving damage tolerant primary composite aircraft structures. In: 11th DoD/FAA/NASA conference on fibrous composites in structural design, Fort Worth, TX, 26-28 August 1996.

6. Boller C, Chang FK and Fijino Y. Encyclopedia of Structural Health Monitoring. Hoboken, NJ: John Wiley \& Sons, 2009.

7. Willberg C, Duczek S, Vivar-Perez JM, et al. Simulation methods for guided wave-based structural health monitoring: a review. Appl Mech Rev 2015; 67(1): 10803-10820.

8. Su Z and Ye L. Identification of damage using LambWaves: from fundamentals to applications. London: Springer, 2009.

9. Yuan S, Ren Y, Qiu L, et al. A multi-response-based wireless impact monitoring network for aircraft composite structures. IEEE Trans Ind Electr 2016; 63(12): 7712-7722.

10. Smithard J, Rajic N, van der Velden S, et al. An advanced multi-sensor acousto-ultrasonic structural health monitoring system: development and aerospace demonstration. Materials 2017; 10(7): 832.

11. Eckstein B and Moix-Bonet M. Lamb wave interaction at delamination and debondings due to impact damage in complex stiffened CFRP structures. ASME J Nondestruct Evaluat 2018; 1: 031003.

12. Willberg C, Koch S, Mook G, et al. Continuous mode conversion of lamb waves in CFRP plates. Smart Mater Struct 2012; 21(7):075022.
13. Kessler SS, Spearing SM and Soutis C. Damage detection in composite materials using lamb wave methods. Smart Mater Struct 2002; 11(2): 269-278.

14. Odinets T. Sizing of essential SHM components of IVHM architecture and computing the relevant costs via scalable simulation, 2017, https://elib.dlr.de/121790/1/ Paper_DLRK.pdf

15. Meyer H. Reduction of uncertainties in SHM data transmission and failure analysis within limitations to economic and safety aspects. In: AST international workshop on aircraft system technologies, Hamburg, 21-22 February 2017.

16. Breguet L. Calcul du poids de combustible consumm par un avion en vol ascendant. Comptes Rendus Hebdomadaires Sances l'Acadmie Sci 1923; 177: 870-872.

17. Hwang I and Lee JS. Buckling of orthotropic plates under various inplane loads. KSCE J Civil Eng 2006; 10(5): 349-356.

18. Adams D. Damage tolerance testing of composites, 2016, https://www.compositesworld.com/articles/damage-tolerancetesting-of-composites(2)

19. Abrate S. Impact on composite structures. Cambridge: Cambridge University Press, 1998.

20. Cantwell WJ and Morton J. The impact resistance of composite materials - a review. Composites 1991; 22(5): 347-362.

21. Mallick PK. Fiber-reinforced composites: materials, manufacturing, and design. 3rd ed. New York: CRC Press, 2007.

22. PCB Piezotronics. MTS System Corporation. Sensors for machinery health monitoring, 2017, https://www.pcb.com.

23. Zhao X, Gao H, Zhang G, et al. Active health monitoring of an aircraft wing with embedded piezoelectric sensor/actuator network: I. Defect detection, localization and growth monitoring. Smart Mater Struct 2007; 16(4): 1208-1217.

24. Flynn EB and Todd MD. Bayesian probabilistic structural modeling for optimal sensor placement in ultrasonic guided wave-based structural health monitoring. In: Smart sensor phenomena, technology, networks, and systems 2010, San Diego, CA, 8-10 March 2010. New York: SPIE.

25. LORD Corporation. Wireless sensor networks: Lord, microstrain sensing systems, 2016, http://www. microstrain.com/

26. Annighoefer B, Ihle $\mathrm{H}$ and Thielecke F. An easy-to-use real-time AFDX simulation framework. In: 2016 IEEE/ AIAA 35th Digital Avionics Systems Conference (DASC), Sacramento, CA, 25-30 September 2016, pp. 1-9. New York: IEEE.

27. Ekman M. KTH royal institute of technology in Stockholm, 2008, https://www.scribd.com/document/347676544/ 3-Ekman-Saab

28. Airbus. A320, aircraft characteristics, airport and maintenance planning: Airbus, 2018, https://www.airbus.com/ content/dam/corporate-topics/publications/backgrounder $\mathrm{s} /$ techdata/aircraft_characteristics/Airbus-Commercial-Ai rcraft-AC-A320-Feb18.pdf 
29. Moir I, Seabridge A and Jukes M. Civil avionics systems, 2nd ed. Hoboken, NJ: John Wiley \& Sons, 2013.

30. Keller KJ, Maggiore J, Safa-Bakhsh R, et al. Sensory Prognostics and Management System (SPMS). Troy, MI: SAE International, 2012.

31. FAA. Aviation maintenance technician handbookairframe, vol. 1. Washington, DC: FAA, 2012.

32. Nexans. Nexans: our products, 2017, https://www. nexans.com
33. Elettrotekkabel S.p.A. Elettrotekkabel group, 2017, http://www.elettrotekkabel.com

34. Schmitt D and Gollnick V. Air transport system. London: Springer-Verlag, 2016.

35. Dollmayer $\mathbf{J}$ and Carl UB. Einfluss des leistungsbedarfs von flugzeugsystemen auf den kraftstoffverbrauch. In: DGLRJT 2004, Dresden, 20-24 September 2004. doi: $10.15480 / 882.212$. 